\title{
A Study on Massive Mimo 5G Challenges
}

\author{
Kanchana Devi $\mathrm{A}^{\mathrm{a}, 1}$ and Bhuvaneswari $\mathrm{B}^{\mathrm{b}}$ \\ ${ }^{a} P G$ Scholar, Dept of ECE, Panimalar Engineering College, Chennai \\ ${ }^{\mathrm{b}}$ Professor, Dept of ECE, Panimalar Engineering College, Chennai
}

\begin{abstract}
Massive MIMO is a advance of MIMO technology. M-MIMO use hundreds of Base station (BS) to simultaneously serve multiple users. It combines with millimeter wave (mmWave) to provide huge spectral efficient, high reliability and high energy efficiency. Massive MIMO gives huge antennas, high signal strength, less noise reduction and also using better channel model. This paper discusses the detail description of fifth generation $(5 \mathrm{G})$ network architecture and to improve massive MIMO in existing technology.
\end{abstract}

Keywords: 5G, Massive MIMO, mmWave, HetNet, QoS, MWT, SDN, SON, mRATs.

\section{Introduction}

In mobile communication system, some drawbacks are occurred in MIMO technology. To overcome this, to go for massive MIMO. It is classified into two types; single user MIMO and multi user MIMO. It is made with an installed dispersed antenna array of approximate hundreds of antenna unit. It can be designed by the large antenna arrays at outside base stations. Hexagonal cell scattered by less number of antenna and connected base station via optical fiber.

In addition, MIMO technology is used to combine with M-MIMO technology. It is used in the indoor wireless network to increase data rate as they have high bandwidth. The advantage of massive MIMO is High spectrum efficiency caused by large multiplexing gain, high reliability caused by large diversity gain. It utilizes beamforming technology, enable that targeted use of spectrum[1].

Massive MIMO and mmWave technologies combined together to work with currently used networks and also support various technologies. mmWave is a very less wavelength. It is possible to pack Huge antennas into a small coverage area with the help of massive MIMO using BSs and UE. Benefits of millimeter waves such as broad bandwidth, high spatial resolution, size and weight of mmwave equipment. Another technology of 5G HetNet based on mmWave and massive MIMO are used. Heterogeneous network provides a more optimized Radio Resource Management and better algorithms to enhance Quality of Service (QoS) requirements [2]. It comprises

\footnotetext{
${ }^{1}$ A.Kanchana Devi, PG Scholar, Dept of ECE, Panimalar Engineering College, Chennai, India; E-mail: akanchanadeviselvi@gmail.com.
} 
Remote Radio Heads and Relays in Wireless Network. The HetNet provides services with massive connectivity, ultra high data rates, ultra low latency, much improved security, very low energy consumption and quality of experience (QoE) [3].

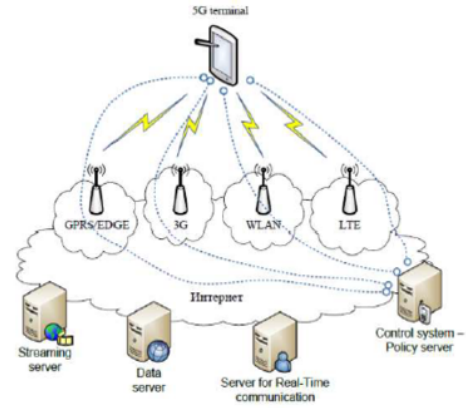

Figure 1. 5G Architecture[4]

Figure 1 describes $5 \mathrm{~g}$ architecture: It is also used to support GPRS, 3G, Wireless LAN, Long time evolution and LTE advance. It worked with adaptive network of architecture. $5 \mathrm{G}$ is evolved from previous generation. These generations are connected to control system policy server, streaming server, data server and server for real time communication[4].

\section{Related Works}

\subsection{Different Layers of $5 G$ Network}

Paul Nikalich et.al[5] discussed various layer architecture of 5G. We will go through the OSI layers such as physical layer, datalink layer and network layer. In $5 \mathrm{G}$ network, physical and datalink layer defined by Open Wireless Architecture.

5G mobile communication, Network layer divided into two categories. There are lower and upper network layer. It supports both IPv4 and IPv6. It provided continues data to user without losses.

5G terminal, the transport layer used to deliver data from BS to user. It performs both transport and session in OSI layer. TCP is used in this protocol. This TCP versions, segments are lost due to congestion. So, It can be retransmitted to complete the transmission session.

In application layer specified to share data to customer in real time application. Qos includes delay, losses, jitter, bandwidth etc. 


\begin{tabular}{|c|}
\hline Application layer \\
\hline Presentation layer \\
\hline Session layer \\
\hline Transport layer \\
\hline Network layer \\
\hline Data link layer \\
\hline Physical layer \\
\hline
\end{tabular}

OSI Stack

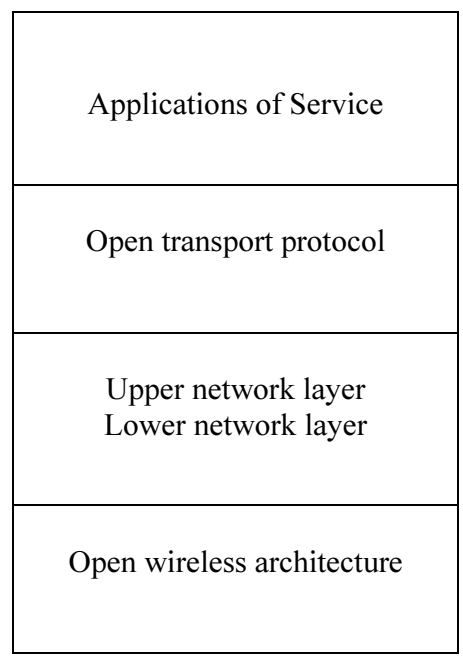

5G Network Stack

Figure 2. Different layer in 5G [5]

\subsection{Enabling Technology}

P. Rost et.al[6] discuss about the important technologies for $5 \mathrm{G}$ :

\subsubsection{Small Cell Deployment (SCD)}

It consists of micro-cells, pico-cells or femto-cells those are called as heterogeneous network (HetNet).

\subsubsection{Machine Type Communication (MTC)}

It is defined machine to machine communication. It enables direct connection of electronic devices. It also needs quite low latency and remote controlling of mobile devices.

\subsubsection{Millimeter Wave Technology (MWT)}

This application consists the band from 30 to $300 \mathrm{GHz}$. In this spectrum, Omnidirectional path loss is increased that can be adjusted through proper beam-forming.

\subsection{Network sharing multi technology}

Figure3 illustrates an example of 5g supporting various technologies: It contains small cells, ultra dense provides Multi RAT and beamforming for high data rate, vehicular communication, sensor networks, machine to machine communication. It also gives to 
perform shared spectrum, direct mode communications, Multihop and Multi eNodeB communications.

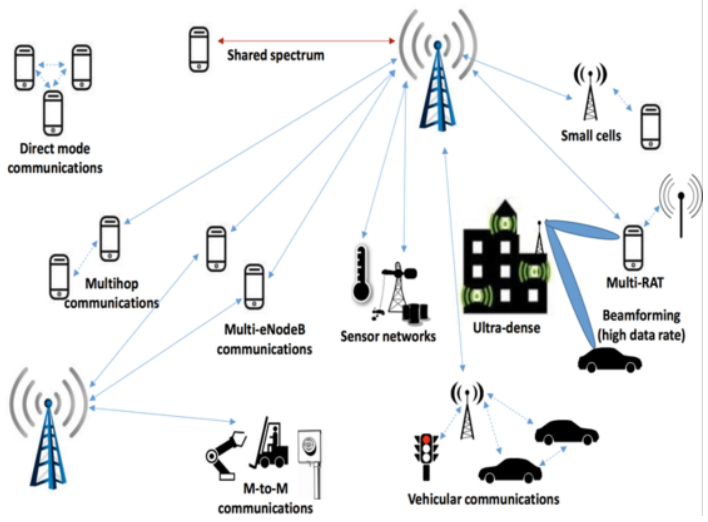

Figure 3. 5G supporting various technologies [7]

\section{$2.45 G$ Design Objectives For M-MIMO}

R. Baldemair et.al[8] tried to solve spectrum lacking, cognitive radio networks is used to increase radio spectrum. This architecture provides a solution for fast processing and flexible arrangement by different layers. The concept of search network, base station working becomes easier and decreased functionalities make it to less energy consumption.

Multi-connectivity is a technology to get the features like data rate, latency, reliability and availability. The network is controlled by device to device [9]. It includes peer to peer, multicast and broadcast communication. Higher rank modulation and coding schemes used to make better spectral efficiency and added with massive MIMO system to get system capacity more.

\subsection{Various Applications Of M-MIMO}

M-MIMO is a current research area of wireless communication. Some areas are explained in this section[10].

\subsubsection{Internet of Things}

5G Networks need to work with Internet of Things and Machine to Machine Communication. Here, Sensors required low data rates but the main challenge is network connectivity. So, traffic occurs in the network. To overcome this we use in M-MIMO[11].

\subsubsection{Vehicular communication Network}

It is used in vehicle. In this communication reduced road accident and to improve traffic rule efficiency. It acts as an intelligent tranportation system. It allows high speed data between two vehicles and reduce noise[12]. 


\subsubsection{Application of Security}

It is more efficient to secure data by using encryption and decryption. It researched in the pilot design, time division duplex systems. Security of physical layer works with DSP processing, Coding and modulation techniques and Multi access scheme[13].

\section{Conclusion}

In this paper discussed the important challenges for $5 \mathrm{G}$ networks which counts higher data rate, higher capacity, low cost and best QoS. The 5G architecture combines various enablers like small cells, massive MIMO and peer-to-peer communication. It maintain data to keep stable and secured in wireless communication. This paper also described different technologies about their drawbacks and how to overcome, which techniques will be enhanced to improve their efficiency.

\section{References}

[1] H. Q. Ngo, E. G. Larsson, and T. L. Marzetta, Energy and spectral efficiency of very large multiuser MIMO systems. IEEE Trans. Commun., vol. 61, no. 4, pp. 1436-1449, Apr. 2013.

[2] T.V. P. Sundararajan, Enhancing QoS parameters of group mobile model in WiMAX networks for military applications. Proceeding IEEE Computer 4th International Conference on Computing, Communications and Networking Technologies (ICCCNT), pp. 1-8, 2013.

[3] J. Noll and M. N. R. Chowdhary .5G: Service continuity in heterogeneous environments. Wireless Personal Communication, vol. - 57, no. - 3, pp. 413-429, 2011.

[4] A. Banchs et al., A Novel Radio Multiservice Adaptive Network Architecture for 5G Networks.Proceeding 81st IEEE VTC, May 2015.

[5] Paul Nikalich, Chih Lin I, Jouni Korhonen, Ragar Marks, Standards for 5G and Beyond: Their use, cases and applications.vol. - 1, no. - 2, June 2017.

[6] P. Rost et al., Mobile Network Architecture Evolution towards 5G.IEEE Communication Magazine, vol. - 54, no. - 5, May 2016.

[7] K. Samdanis, X. Costa Perez and V. Sciancalepore, From Network Sharing to Multi-Tenancy: The 5G Network Slice Broker.IEEE Communication Magazine, vol. - 54, no. - 7, July 2016.

[8] R. Baldemair et al., Evolving wireless communications: Addressing the challenges and expectations of the future.IEEE Vehicular Technology Magazine, vol. - 8, pp. 24-30, Mar. 2013.

[9] A. Asadi, Q. Wang, and V. Mancuso, A survey on device-to-device communication in cellular networks .IEEE Commun. Surveys Tuts., vol. 16, no. 4, pp. 1801-1819, Fourth Quart. 2014.

[10] A. Checko et al., Cloud ran for mobile networks-A technology overview. IEEE Commun. Surveys Tuts., vol. 17, no. 1, pp. 405-426, First Quart. 2015.

[11] J. Lin, W. Yu, N. Zhang, X. Yang, H. Zhang, and W. Zhao, A survey on Internet of Things: Architecture, enabling technologies, security, privacy, and applications. IEEE Internet Things J., vol. 4, no. 5, pp. 1125-1142, Oct. 2017.

[12] D. Gesbert, S. Hanly, H. Huang, and S. Shamai Shitz, Multi-cell MIMO cooperative networks: A new look at interference.IEEE J. Sel. Areas Commun., vol. 28, no. 9, pp. 1380-1408, Oct. 2010

[13] B. Wong, I. Stoyanov, and E. Sirer, Octant: A comprehensive framework for the geolocalization of Internet hosts.in Proc. NSDI, 2007, vol. 7, p. 23. 